\title{
Problems of Remote Temperature Measurement of Small Objects of Electricity Power Systems-On the Example of Lashing Clamps of Bridge Connections on High Voltage Poles
}

\author{
Waldemar Minkina
}

Citation: Minkina, W. Problems of Remote Temperature Measurement of Small Objects of Electricity Power Systems-On the Example of Lashing Clamps of Bridge Connections on High Voltage Poles. Energies 2021, 14, 5041. https://doi.org/10.3390/ en14165041

Academic Editor: Hugo Morais

Received: 20 July 2021

Accepted: 15 August 2021

Published: 17 August 2021

Publisher's Note: MDPI stays neutral with regard to jurisdictional claims in published maps and institutional affiliations.

Copyright: (C) 2021 by the author. Licensee MDPI, Basel, Switzerland. This article is an open access article distributed under the terms and conditions of the Creative Commons Attribution (CC BY) license (https:/ / creativecommons.org/licenses/by/ $4.0 /)$.
Faculty of Electrical Engineering, Czestochowa University of Technology, Al. Armii Krajowej 17, PL 42-200 Częstochowa, Poland; waldemar.minkina@pcz.pl; Tel.: +48-34-608-018-803

\begin{abstract}
The article presents problems occurring during remote temperature measurement of lashing clamps of bridge connections on high voltage poles using thermal imaging cameras. The basic metrological parameters of thermal imaging cameras are described. On this basis, typical errors made during the inspection of high voltage lines supplying power substations are presented using infrared cameras. Researching the possible solutions for the problems of remote temperature measurement of small objects of electricity power systems-on the example of lashing clamps of bridge connections on high voltage poles in the proposed paper and showing the basic metrological aspects and parameters of thermal imaging cameras are important because, in this way, it is observed to eliminate costly interruptions in the supply of electricity associated with the breaking of power lines. Small objects are quite difficult to be controlled and monitored on large grids and on large powerline poles; thus, it is very challenging to interpret the data offered by thermograms. The problem of remote temperature measurement of small objects in electrical power engineering is very important from the point of view of the quality and reliability of electricity supply. Obtaining early warning information about the occurrence of overheating, e.g., on lashing clamps of bridge connections, is very important, as it eliminates costly interruptions in the supply of electricity associated with the breaking of power lines supplying high voltage switchgears or substations.
\end{abstract}

Keywords: remote temperature measurement; thermovision measurements; lashing clamps of bridge connections; thermal imaging cameras

\section{Introduction}

The problem of remote temperature measurement of small objects in electrical power engineering is very important from the point of view of the quality and reliability of electricity supply. Obtaining early warning information about the occurrence of overheating, e.g., on lashing clamps of bridge connections, is very important, as it eliminates costly interruptions in the supply of electricity associated with the breaking of power lines supplying high voltage switchgears or substations.

The only modern tool for such diagnostics is a thermal imaging camera, which allows remote temperature measurement of these objects. It must be emphasised that a lashing clamp on a high voltage line is a small object. Therefore, in order to correctly measure the temperature of such an object, it is necessary to possess adequate knowledge and experience regarding the metrological capabilities of a thermal imaging camera when remotely measuring the temperature of a small object.

This article describes the basic metrological parameters of a thermal imaging camera, important in the interpretation of the remote temperature measurement of small objects. Based on this knowledge, a practical example is provided as to how to correctly remotely measure the temperature of a lashing clamp of bridge connections of a power line. A case of incorrect and correct remote temperature measurement of this small object is described, showing, at the same time, the differences in the readings for both cases. 
This is an important case concerning the practice of non-contact temperature measurement of power line elements of small size and the interpretation of the recorded thermograms [1-10].

For this purpose, at the beginning of the article, the basic metrological parameters of observational and measurement thermal cameras are described.

\section{Basic Metrological Parameters of Observation and Measurement Thermal Imaging Cameras}

Thermal cameras (observation and measurement) are characterised by a number of parameters defining their observational characteristics and metrological properties. A few of them are described below. The knowledge of these parameters is essential for the correct interpretation of thermograms obtained from thermovision measurements of small objects in electricity power systems.

The MDTD and MRTD characteristics are the basis for most models of evaluating the observational quality of thermal imaging devices. Sometimes, these characteristics are abbreviated as MDT and MRT.

MDTD-(minimum detectable temperature difference) describes the ability of an observational thermal imaging camera to detect objects of small angular size against a homogeneous background. It is defined as the dependence of the minimum difference between the temperature of a square (or circular) $T_{o b}$ test and the temperature of a homogeneous background $T_{0}$, enabling detection of the test by an experienced observer on the camera monitor, on the inverse of the characteristic angular dimension of the test [5-7]. In practice, a homogeneous background can be, for example, the sky or a body of water. Figure 1 shows an example plot of the MDTD characteristic with the relative dimensions of the test are, marked $F$. It can be observed that this characteristic has no asymptote. This means that an object of any small angular size can be detected, provided that it has a sufficiently high temperature.

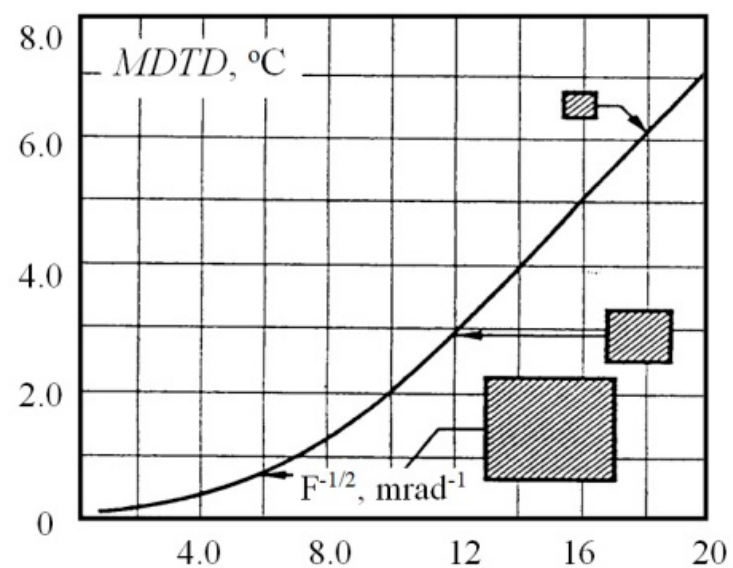

Figure 1. Example plot of MDTD characteristics with the relative dimensions of the test area, marked $F[5-7]$.

MRTD-(minimum resolvable temperature difference) is used to assess the performance of observation cameras. It is defined as the dependence of the minimum temperature difference between the temperature of a four-strip test $T_{o b}$ and the background temperature $T_{0}$, at which an experienced observer can distinguish all the test strips on the camera monitor, on the spatial frequency of the test $f$. MRTD relates temperature resolution to camera spatial resolution and describes the effect of noise on these parameters. Knowledge of this characteristic makes it possible to determine the probabilities of detection, recognition and identification of an object against a non-uniform background. In practice, heterogeneous backgrounds can be, e.g., ground objects. Figure 2a shows an example plot of the MRTD characteristics with the relative dimensions of the four-band test marked, and Figure $2 b$ 
shows an example of a test with normalised dimensions. It can be observed that, unlike the MDTD characteristic, the MRTD characteristic has a vertical asymptote for large values of the test spatial frequencies $f$. This is due to the limited spatial resolving capacity of the camera, determined by the modulation transfer function (MTF), described later in this article. The spatial frequency $f$ is, in this case, expressed in cycles of the test per milliradian. One cycle refers to the strip and the free space between the strips.

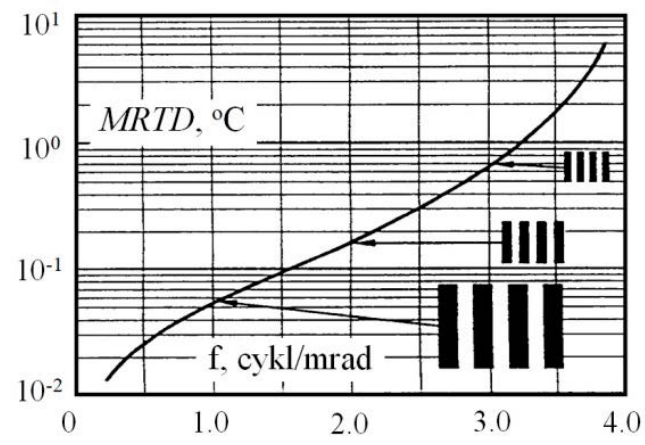

(a)

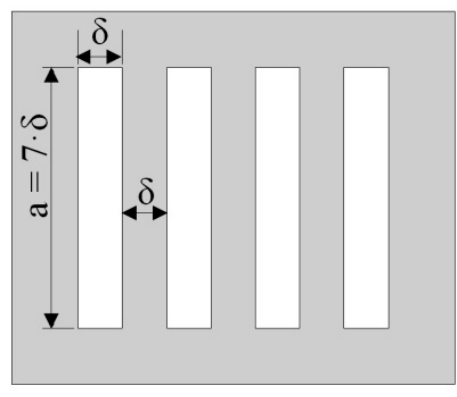

(b)

Figure 2. (a) Example plot of $M R T D$ characteristics ( $f$, spatial frequency of the test); (b) example appearance of a standardised four-band test for MRTD determination with marked dimensions [5-7].

Typical measurement sets for the study of the discussed characteristics consist of three basic blocks: a blackbody, a rotating disc with four-band or circular tests and an infrared collimator. The method of determining MDTD characteristics is similar to that of MRTD. The difference concerns the shape of the test, as shown in Figures 1 and 2b [5-7].

The special feature of MDTD and MRTD characteristics is the fact that they depend on the subjective assessment of the observer. The detailed procedure of their determination can be found, among others, in the works [5-7] and in the American standards [8-10].

MTF-(modulation transfer function) is a function determined by theoretical models. It defines the spatial resolution of a thermal camera as a function of distance from the object. It is a basic function in models describing MRTD characteristics [5-7].

The basic metrological parameters of measurement thermal cameras are described below. The knowledge of these parameters is necessary for the correct interpretation of thermograms obtained from thermovision measurements of small electrical power facilities.

\section{Basic Metrological Parameters of Measuring Thermal Imaging Cameras}

NETD - (noise equivalent temperature difference) defines the difference between the temperature of the test object and the environment causing the generation of a signal equal to the noise [11-14]. It is called the temperature resolving power, or briefly, the temperature resolution. It is defined as the quotient of the rms value of the noise voltage $U_{n}$ and the ratio of the signal voltage increment $\Delta U_{s}$ to the small increment of the temperature difference between the technical blackbody (or test) $T_{o b}$ measurement field and the background $T_{0}$ :

$$
N E T D=\frac{U_{n}}{\frac{\Delta U_{s}}{T_{o b}-T_{o}}}=\frac{T_{o b}-T_{o}}{\frac{\Delta U_{s}}{U_{n}}} .
$$

The temperature of the measuring field of a technical black body is usually $T_{o b}=30{ }^{\circ} \mathrm{C}$ of the background $T_{o}=22{ }^{\circ} \mathrm{C}$, while the difference $T_{o b}-T_{o}$ should be within the limits of $5 \div 10 \mathrm{~K}$, as shown in Figure 3 . 


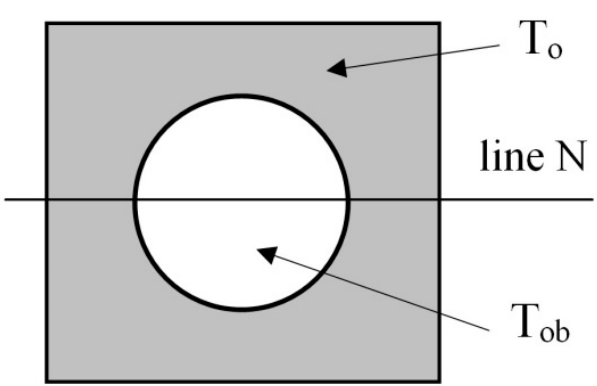

(a)

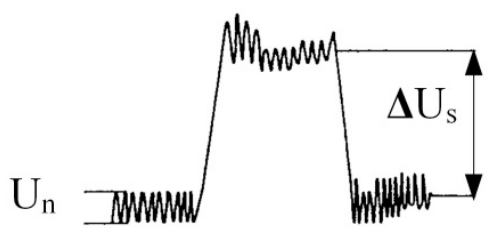

(b)

Figure 3. Interpretation of the determination of the NETD parameter [11-15]. (a) situation when the temperature Tob approximately equal to the background temperature To; (b) the observable Us signal from the $\mathrm{N}$-line detector.

There is also a slightly different definition of this parameter. NETD is defined as the difference in temperature between $T_{o b}$ and $T_{0}$, which the detector 'looks at', resulting in a signal change at its output equal to the detector noise. When determining this parameter, a technical blackbody measuring field with a temperature $T_{o b}$ approximately equal to the background temperature $T_{o}$ is observed, as depicted in Figure 3a. The observable $U_{s}$ signal from the N-line detector is shown in Figure 3b. NETD is determined when the value of the signal $U_{s}$ is equal to the value of the noise signal $U_{n}$.

In both cases, NETD defines what minimum increment of temperature difference or what smallest temperature difference $T_{o b}$ and $T_{o}$ can distinguish a single detector (ruler or detector array) for a given amplifier bandwidth. According to the theory given in [11], narrowing the amplifier bandwidth reduces the noise voltage, i.e., allows the value of the NETD parameter to reduce, but this carries with it a deterioration of the spatial resolution, e.g., at a constant scan rate. In measurements, instead of a technical blackbody measurement field, circular or rectangular tests with a stabilised $T_{o b}$ temperature can be used. The NETD definitions given do not take into account the size of the object, the physiology of human perception or the properties of the display system [16-18].

Example $[12,14,17,18]$

The catalogues provide the following NETD values of the two cameras:

- $\quad N E T D_{1}=0.1 \mathrm{~K}$ for $T_{o b 1}=30^{\circ} \mathrm{C}$;

- $\quad N E T D_{2}=0.2 \mathrm{~K}$ for $T_{o b 2}=50^{\circ} \mathrm{C}$.

How does one determine which system has the lower value of noise voltage $U_{n}$ ? For both cameras, assume the same value of the voltage increment of the detector signal $\Delta U_{s}$. The value of $T_{0}$ in both cases was $22^{\circ} \mathrm{C}$.

According to Formula (1), it is obtained:

$$
U_{n}=\frac{N E T D}{T_{o b}-T_{o}} \Delta U_{s}
$$

$\begin{aligned} & U_{n 1}=\frac{N E T D_{1}}{T_{p h}-T_{o}} \Delta U_{s}=\frac{0,1 \Delta U_{s}}{8}=0,0125 \cdot \Delta U_{s} \\ & \text { i.e., } \quad U_{n 2}=\frac{R E T D_{2}}{T_{o b 2}-T_{o}} \Delta U_{s}=\frac{0,2 \Delta U_{s}}{28}=0,007 \cdot \Delta U_{s} .\end{aligned}$

From the above, it can be observed that the second camera has a lower noise voltage value despite the fact that $N E T D_{2}>N E T D_{1}$.

A higher NETD value indicates a lower camera sensitivity. This is why the NETD parameter is called thermal sensitivity or temperature resolution in thermal camera catalogues. When specifying the catalogue value of the temperature resolution of the camera, it is necessary to additionally specify the $T_{o b}$ value at which this parameter was determined (Figure 4). 


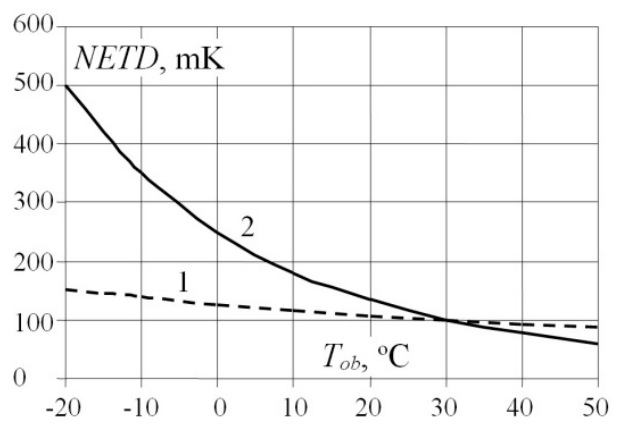

(a)

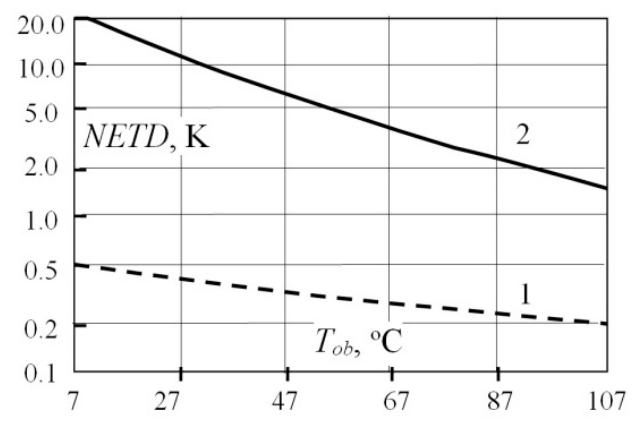

(b)

Figure 4. Example charts of the temperature resolution dependence of NETD on $T_{o b}$ of the following cameras: (a) longwave LW-1 and shortwave SW-2 [12]; (b) inframetrics 760 BB with filters, working in the longwave LW, $8 \div 12 \mu \mathrm{m}-1$ and shortwave, SW $3 \div 5 \mu \mathrm{m}-2[5]$.

A typical value of the NETD temperature resolution is:

- $10 \div 30 \mathrm{mK}$, for QWIP detector cameras for scientific research;

- $50 \div 100 \mathrm{mK}$, for measuring cameras;

- $\quad>200 \mathrm{mK}$, for observation cameras.

The procedure of determining NETD is not yet standardised, and prospectuses provide different values of this parameter that are determined in different ways. This may lead to a situation when in the prospectus, an inferior camera is characterised by a better NETD parameter. This parameter is, in fact, one of the most frequently used parameters to assess the metrological properties of thermal imaging cameras.

\section{Problems of Remote Temperature Measurement of Small Objects of Electricity Power Systems-On the Example of Lashing Clamps of Bridge Connections on High Voltage Poles}

FOV - (field of view) determines the area that can be observed from a given distance $\mathrm{d}$ by the optics installed in the camera. This parameter defines the spatial (geometric) resolution of a thermal imaging camera. FOV is specified in metres and refers to the horizontal $(H$, horizontal) and vertical $(V$, vertical) directions of observation. Table 1 provides example values of the field of view for $24^{\circ} \times 18^{\circ}$ optics, depending on the distance. For the optics given above, the field of view can be calculated from the formulas:

$$
H=d \cdot \sin \left(24^{\circ}\right), \quad V=d \cdot \sin \left(18^{\circ}\right) .
$$

Table 1. Field of view (FOV) values for $24^{\circ} \times 18^{\circ}$ optics.

\begin{tabular}{|c|c|c|c|c|c|c|c|}
\hline$d, \mathrm{~m}$ & 0.50 & 1.0 & 2.0 & 5.0 & 10 & 30 & 100 \\
\hline$H, \mathrm{~m}$ & 0.20 & 0.41 & 0.81 & 2.0 & 4.1 & 12 & 41 \\
\hline$V, \mathrm{~m}$ & 0.15 & 0.31 & 0.62 & 1.5 & 3.1 & 9.3 & 31 \\
\hline
\end{tabular}

IFOV - (instantaneous field of view) is the inverse of the so-called distance coefficient $(d / D$, distance to spot size ratio) and determines what area constitutes the field of view of a single detector (pixel) of the matrix. Therefore, from a practical point of view, it should be called the 'required' or 'minimum' field of view. This parameter defines the spatial (geometric) resolution of a measuring thermal camera. In short, in catalogues it is called spatial resolution. For example, for $24^{\circ} \times 18^{\circ}$ optics, from a distance of $1 \mathrm{~m}$, the $H \times$ $V$ field of view provided in Table 1 is $0.41 \times 0.31 \mathrm{~m}$. Assuming that the camera has a matrix, for example, consisting of $320 \times 240$ detectors, the field of view of a single detector $H_{\min } \times V_{\min }$ will be:

$$
\frac{0.41}{320}=\frac{0.31}{240}=1.3 \mathrm{~mm} \cdot 1.3 \mathrm{~mm} .
$$


This means that with a camera with $24^{\circ} \times 18^{\circ}$ optics equipped with a $320 \times 240$ sensor array, an 'overheat' of $1.3 \mathrm{~mm} \times 1.3 \mathrm{~mm}$ can be detected from a distance of $1 \mathrm{~m}$. Of course, overheating can also be detected over a smaller area, but the measured temperature will be significantly underestimated. The area increases and decreases in proportion to the distance $d$. For example, for $d=10 \mathrm{~m}$, it is $13 \mathrm{~mm} \times 13 \mathrm{~mm}$.

Another way to calculate the IFOV is to calculate in radians the angle $\alpha_{\text {rad }}$ of the optics opening for a single detector:

$$
\begin{aligned}
& \alpha_{\text {rad } H}=\frac{24 \pi}{180 \cdot 320}=0.0013 \mathrm{rad} \text { thereby } H_{\text {min }}=d \cdot \sin (0.0013)=1.3 \mathrm{~mm}, \\
& \alpha_{\text {radV }}=\frac{18 \pi}{180 \cdot 240}=0.0013 \mathrm{rad} \text { thereby } V_{\text {min }}=d \cdot \sin (0.0013)=1.3 \mathrm{~mm} .
\end{aligned}
$$

In other words, the IFOV is the area that, through the optics, a single pixel of the matrix 'looks at' and defines the absolute lower limit of the size of the measured object. The spatial resolution of the camera depends on the opening angle of the optics used and the number of detectors (pixels) in the matrix. The smaller the angle of the optics and the more detectors the matrix has, the better the spatial resolution of the camera; thus, smaller objects can be observed. However, there are obvious limitations associated with increasing the number of detectors in the matrix and building lenses with a smaller angle, i.e., smaller field of view.

Camera manufacturers usually specify the spatial resolution in milliradians [mrad]. For example, in brochures, you can read that for $24^{\circ} \times 18^{\circ}$ optics, the spatial resolution is $1.3 \mathrm{mrad}$. This parameter means the value of the IFOV field of view for a single detector (pixel) of the matrix $H_{\text {min }}$ and $V_{\text {min }}$, calculated according to Formula (4).

The described situation can be further explained by analysing Figure 5. In Figure 5a,b, two cases of detector exposure by a small object are presented. In Figure 5b, the object is accidentally positioned so that it completely exposes at least one detector of the array, while in Figure 5a, the same object is not able to completely expose any detector. From Figure 5d, it can be observed that the temperature of the extraction holder measured from close-up is higher than the temperature of the same holder measured from far away (Figure 5c). This is due to the fact that at close range, the camera optics guarantee full illumination of at least one detector of the camera array. If the object is warmer than the environment behind it, as in Figure 5a, the indicated temperature will be underestimated when the detector is not fully illuminated; otherwise, it would be overestimated. The above description may explain the English term for this parameter as an instantaneous field of view. This means that, theoretically, only at a certain moment in time, for ideal electronics and optics, meeting the requirements of the IFOV parameter may be sufficient for the correct illumination of the detector. In the time domain, the term instantaneous means the instantaneous value of the signal in time, while in the spatial domain, it means the response of the detector to the irradiance of a given point of the object.

In Figure 5c,d marked, respectively:

- IRmax - the maximum temperature across the entire surface of the thermogram, as determined by the maximum temperature selected from all the detectors in the array;

- ARmax - the maximum temperature across the marked area, as determined by the maximum temperature selected from the detectors illuminated in the area.

It can also be observed in Figure 5c,d that IRmax is equal to ARmax, which further confirms that the location of the maximum temperatures is correct. Analysing Figure 5b, one may come to the conclusion that in order to carry out a correct spot temperature measurement, it is sufficient for an object to expose $2 \times 2$ detectors of the matrix. In practice, however, this is not the case, if only because the object may not have a square or rectangular shape. In addition, all optics distort the image. This is characterised by the occurrence of chromatic aberration, spherical aberration or many other imperfections, as 
shown in Figure $6 \mathrm{~b}$. This parameter is further described by the PSF (Point Spread Function). One of its models is described by the formula:

$$
\operatorname{PSF}(x, y)=\exp \left(-\frac{x^{2}+y^{2}}{2 \sigma^{2}}\right),
$$

where:

$\sigma$ is the parameter defining point response of the optical system (spatial resolution) expressed in milliradians. Its exemplary values may be:

- $\sigma=0.5 \mathrm{mrad}$ for measurement cameras, with better spatial resolution;

- $\sigma=1.0 \mathrm{mrad}$ for observation (imaging) cameras, with poorer spatial resolution.

a)

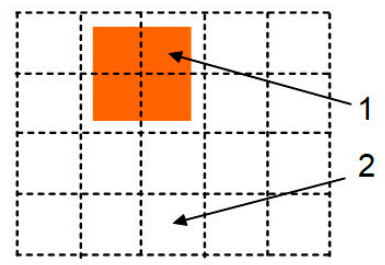

b)
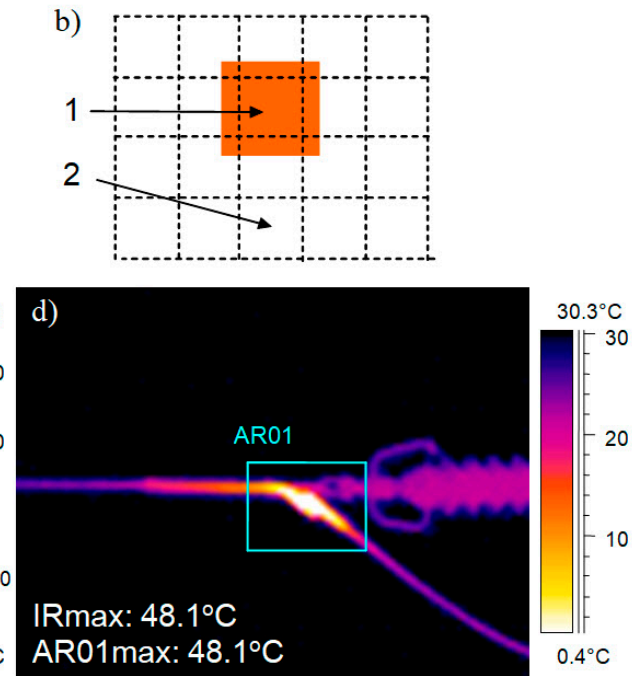

IRmax: $24.2^{\circ} \mathrm{C}$

AR01max: $24.2^{\circ} \mathrm{C}$
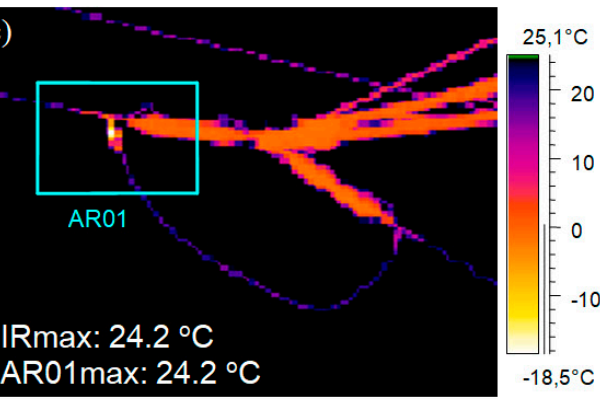

Figure 5. Determination of the minimum size of a small object using the example of lashing clamps of bridge connections of a high voltage power line pole; the position of the object is in relation to the detector array (a) that does not guarantee full illumination of any detector and (b) that guarantees full illumination of at least one detector: 1 , object; 2 , detectors of the matrix; (c) the temperature value of the holder measured from far away, i.e., about 40 metres (electronic 'zoom' optical and digital); (d) the temperature value of the same holder measured from close-up, i.e., about 7 metres (only 'zoom' digital thermogram).
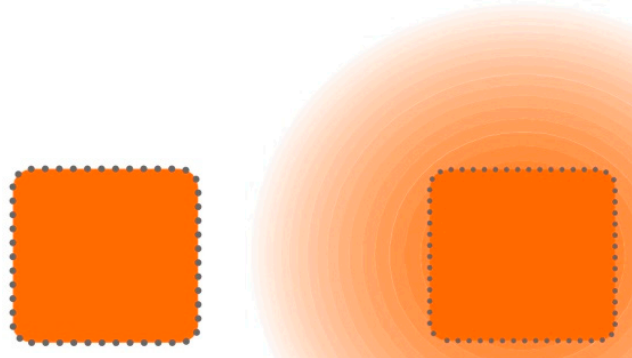

(a)

(b)

Figure 6. Determination of measurement area size: (a) ideal optics, $2 \times 2$ array detectors required; (b) real optics, $3 \times 3$ or $4 \times 4$ (sometimes even $5 \times 5$ ) detectors required [12,19-21].

A graphical illustration of the PSF function is shown in Figure 7. 


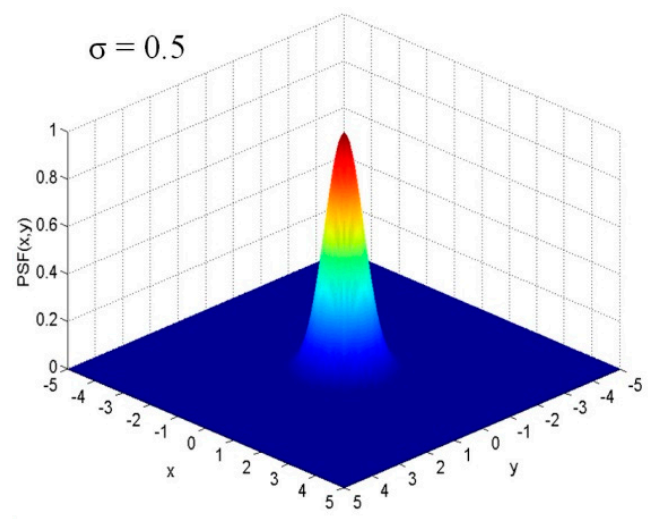

(a)

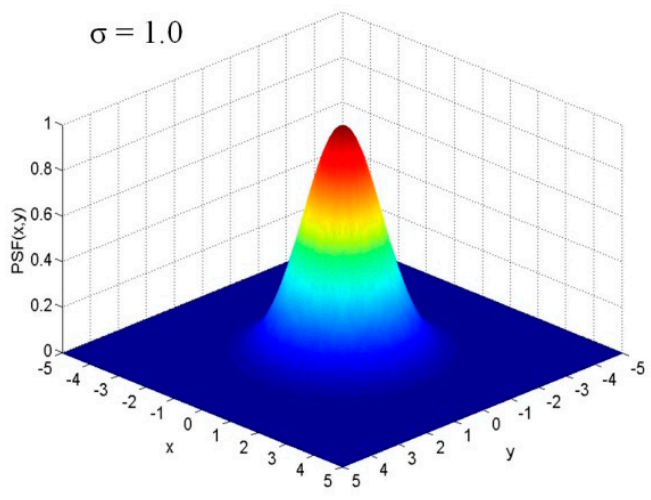

(b)

Figure 7. Explanation of the PSF function of the camera optical system response to a point source of radiation (a) $\sigma=0.5$ mrad for measurement cameras, with better spatial resolution; (b) $\sigma=1.0 \mathrm{mrad}$ for observation (imaging) cameras, with poorer spatial resolution.

In order to avoid the problems described above, it is assumed that the minimum object size should guarantee full illumination of at least $3 \times 3$ or $4 \times 4$ and sometimes even $5 \times 5$ detectors of the array, i.e., it should be between $3 \times 3$ and $5 \times 5$ IFOV for correct temperature measurement.

SRF-(Slit Response Function), similar to the IFOV parameter, describes the ability of a thermal imaging camera with an array detector to measure the temperature of small objects. Figure 8 shows three situations for measuring the temperature of a black body $T_{o b}$ on whose surface the IFOV symbol indicates the field 'seen' by a single array detector. The temperature of $T_{o b}$ corresponds to the $u_{o b}$ signal from the detector. The measurement field of the blackbody is gradually covered by the aperture with temperature $T_{0}$, which corresponds to the signal $u_{o}$ from the detector. As the size $\delta$ of the aperture decreases, the value of the $u_{o b}$ signal changes.
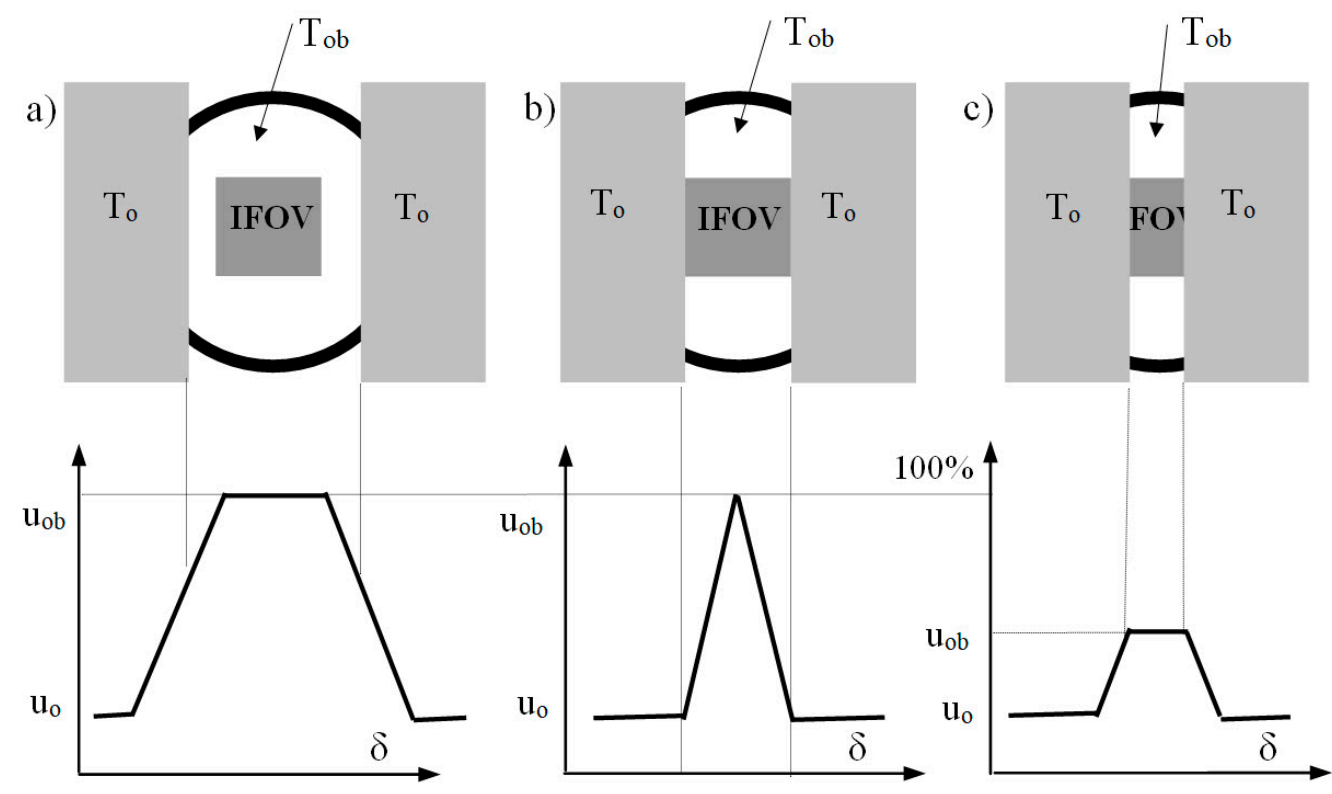

Figure 8. Modulation characteristics of the $u_{o b}$ signal from the array detector depending on the slit width $\delta$ through which an object of IFOV size is observed [12]. The measurement field of the blackbody is gradually covered by the aperture with temperature $T_{0}$, which corresponds to the signal $u_{o}$ from the detector-Figures $(\mathbf{a}-\mathbf{c})$. As the size $\delta$ of the aperture decreases, the value of the $u_{o b}$ signal changes. 
Figure 9 shows the left parts of the modulation characteristics from Figure 8 as a function of the optics opening angle $\alpha_{\text {rad }}$ for a single detector, defined by Formulas (4). Approximately, the value of $\alpha_{\text {rad }}$ also corresponds to the quotient of the slit width $\delta$ and the camera-object distance $d$. Characteristic 1 of Figure 9a concerns the theoretical (idealised) case, while curve 2 concerns the real case, related to the fact that the camera optics and electronics are not ideal. In Figure 9b, we compare real characteristics of modulation of the measurement cameras (curves 1 and 2) and the observation camera (curve 3).

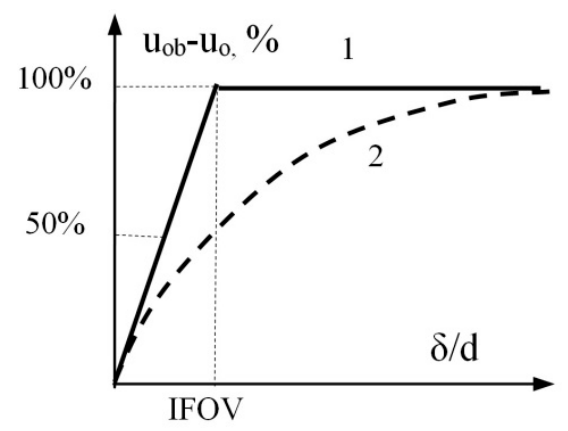

(a)

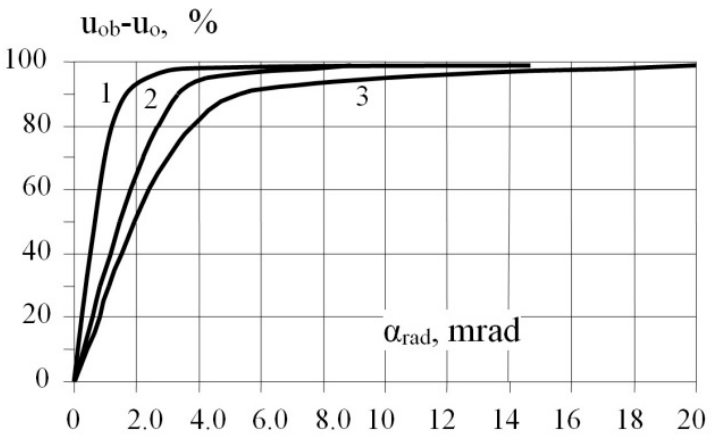

(b)

Figure 9. Modulation characteristics of the $u_{o b}-u_{o}$ signal from the array detector depending on the angle $\alpha_{\text {rad }}$ of optics opening for a single detector: (a) generalisation: 1 , theoretical, idealised case; 2, real camera; (b) practical cases: 1, 2, measurement camera; 3, observation camera [19-21].

Analysing the characteristics from Figure 9b, we can see that the value of the angle $\alpha_{\text {rad }}$ corresponding, e.g., to a 50\% modulation for the measurement camera, is much smaller than for the observation camera. Taking into account the remarks provided during the discussion of the IFOV parameter, it can be concluded that the most accurate measurements are obtained if the modulation value is as high as possible. For example, if the modulation value is $90 \%$, the detectors may receive $10 \%$ too little illumination, i.e., the indicated temperature will be, for example, underestimated by $10 \%$. This is a very large, unacceptable error value. Therefore, a modulation value greater than $98 \%$ should be sought. In Figure $9 b$, the characteristics of the 1,2 measurement cameras correspond to $\alpha_{\text {rad }}=5 \div 8$ mrad, while the characteristic of the 3 observation camera is $\alpha_{\text {rad }}=15 \div 20 \mathrm{mrad}$.

Analysing the above, it can be seen that the modulation characteristics of the slit function $S R F$ confirm the previously stated requirement that in order to guarantee correct measurement with a thermal imaging camera of a certain spatial resolution, characterised by the IFOV parameter, the required object size should be from $3 \times 3$ to $5 \times 5$ IFOV.

\section{Conclusions}

In conclusion, it should be stated that the problem of measuring the temperature of extraction handles of bridge connections (lashing clamps) on high voltage poles is very important from the point of view of the reliability of electricity supply. Obtaining early information about the occurrence of overheating on such a connection is very important, as it eliminates costly interruptions in the supply of electricity associated with the rupture of power lines supplying high voltage switching stations or substations.

The only modern tool for such diagnostics is a thermal imaging camera, which allows remote temperature measurement of these objects. It must be emphasised that a bridge lashing clamp on a high voltage line is a small object. Therefore, in order to correctly measure the temperature of such an object, it is necessary to possess adequate knowledge and experience regarding the metrological capabilities of a thermal imaging camera when remotely measuring this small object.

This article described the basic metrological parameters of a thermal imaging camera that are important in the interpretation of the remote temperature measurement of small objects. Based on this knowledge, a practical example was provided as to how to correctly remotely measure the temperature of a lashing clamp for bridge connections of a high 
voltage power line. A case of incorrect and correct remote temperature measurement of this small object was described, showing, at the same time, the differences in the readings for both cases.

This is an important case concerning the practice of non-contact temperature measurement of power line objects of small size and the interpretation of the recorded thermograms.

It should be emphasised that incorrect interpretation of thermograms obtained from remote thermovision measurements can lead to unnecessary disconnections of power lines and associated unnecessary costs or delayed disconnections of power lines and high costs associated with line breaking and power outages.

For example, if an inexperienced person interprets the thermogram provided in Figure 5, they may make a significant error because the actual temperature of the lashing clamps of bridge connections is about $48^{\circ} \mathrm{C}$, not $24^{\circ} \mathrm{C}$.

Funding: This research received no external funding.

Institutional Review Board Statement: Not applicable.

Informed Consent Statement: Not applicable.

Data Availability Statement: I exclude this statement because the study did not report any data.

Conflicts of Interest: The author declares no conflict of interest.

\section{References}

1. Hulewicz, A.; Dziarski, K.; Dombek, G. The solution for the thermographic measurement of the temperature of a small object. Sensors 2021, 21, 5000. [CrossRef] [PubMed]

2. Sanda, M.; Kojima, T.; Higashi, E.; Maruyama, T.; Iwama, N.; Saka, O. Overhead Transmission Line Monitoring System for Dynamic Rating. SEI Tech. Rev. 2018, 87, 64-69.

3. Ivanov, G.V.; Ivanov, V.G. Temperature and Emissivity Determination of Small-Size Long-Range Object's Using Staring Thermovision Cameras. Infrared Phys. Technol. 2013, 60, 161-165. [CrossRef]

4. Available online: https://www.metronic.dk/CustomerData/Files/Folders/4-download-pdf/13_ir-basics.pdf (accessed on 11 August 2021).

5. Chrzanowski, K. Non-Contact Thermometry-Measurement Errors; SPIE (Society of Photooptical Instrumentation Engineers): Warszawa, Poland, 2000; Available online: https:/ /inframet.com/Literature/Non-contact_thermometry-Measurement\%20errors. pdf (accessed on 16 July 2021).

6. Hartmann, J.; Gutschwager, B.; Fischer, J.; Hollandt, J. Calibration of thermal cameras for temperature measurements using blackbody radiation in the temperature range $-60{ }^{\circ} \mathrm{C}$ up to $3000{ }^{\circ} \mathrm{C}$. In Proceedings of the 7 th International Conference on Infrared Sensors \& Systems 2002, (IRS2'2002), Erfurt, Germany, 14-16 May 2002; Gerlach, G., Ed.; pp. 119-124.

7. Madura, H. Modelling and Testing of Infrared Detection Devices. Habilitation Monograph; WAT Publishing House: Warszawa, Poland, 1998. (In Polish)

8. American Standards: ASTM E 1213 Minimum Resolvable Temperature Difference for Thermal Imaging Systems (MRTD). Available online: https://www.astm.org/DATABASE.CART/HISTORICAL/E1213-14.htm (accessed on 14 August 2021).

9. American Standards: ASTM E 1311 Minimum Detectable Temperature Difference for Thermal Imaging Systems (MDTD). Available online: https:/ / www.astm.org/DATABASE.CART/HISTORICAL/E1311-14.htm (accessed on 14 August 2021).

10. American Standards: ASTM E 1316 Section J. Terms. Available online: https://www.scribd.com/document/325357642/ASTME1316-02a-Standard-Terminology-for-Nondestructive-Examinations-pdf (accessed on 14 August 2021).

11. Nowakowski, A. Advances in Infrared Thermography—Medical Applications; Publishing House Wydawnictwo Gdańskie: Gdańsk, Poland, 2001; ISBN 83-88836-30-7. (In Polish)

12. IR-Book. FLIR Training Materials; Level II (Infrared Training Center-International, itc-i); FLIR Systems AB: Danderyd, Sweden, 2015.

13. Vollmer, M.; Möllmann, K.-P. Infrared Thermal Imaging: Fundamentals, Research and Applications, 2nd ed.; WILEY-VCH Verlag GmbH \& Co. KGaA, 2018; Available online: https:// onlinelibrary.wiley.com/doi/book/10.1002/9783527693306 (accessed on 19 June 2021).

14. Minkina, W.; Dudzik, S. Infrared Thermography_Errors and Uncertainties; John Wiley \& Sons Ltd., Wiley—Blackwell: Chichester, UK, 2009; ISBN 978-0-470-74718-6. Available online: https:/ /www.wiley.com/en-us/Infrared+Thermography:+Errors+and+ Uncertainties-p-9780470682241 (accessed on 16 July 2021).

15. Minkina, W.; Dudzik, S. Simulation analysis of uncertainty of infrared camera measurement and processing path. Measurement 2006, 39, 758-763. [CrossRef]

16. Dudzik, S.; Minkina, W. Application of the numerical method for the propagation of distributions to the calculation of coverage intervals in the thermovision measurements. In Proceedings of the 9th International Conference on Quantitative Infrared Thermography (QIRT’2008), Kraków, Poland, 2-5 July 2008; pp. 179-183, ISBN 978-83-908655-1-5. [CrossRef] 
17. Minkina, W.; Dudzik, S.; Gryś, S. Errors of Thermographic Measurements-Exercises. In Proceedings of the 10th International Conference on Quantitative Infrared Thermography (QIRT'2010), Quebec City, QC, Canada, 27-30 July 2010; pp. 503-509, ISBN 978-2-9809199-1-6. [CrossRef]

18. Minkina, W.; Klecha, D. Atmospheric transmission coefficient modelling in the infrared for thermovision measurements. J. Sens. Sens. Syst. 2016, 5, 17-23. [CrossRef]

19. Danjoux, R. The evolution in spatial resolution. Inframation Mag. 2001, 2, 1-3.

20. Więcek, B.; De Mey, G. Infrared Thermal Imaging_Fundamentals and Application; PAK Publishing House: Warszawa, Poland, 2011; ISBN 978-83-926319-7-2. (In Polish)

21. Budzier, H.; Gerlach, G. Thermal Infrared Sensors-Theory, Optimization and Practice; John Wiley \& Sons Ltd., Wiley-Blackwell: Chichester, UK, 2011; ISBN 978-0-470-87192-8. Available online: https:/ /www.wiley.com/en-us/Thermal+Infrared+Sensors\% 3A+Theory\%2C+Optimisation+and+Practice-p-9780470976753 (accessed on 16 July 2021). 